\title{
Puissance de l'écriture fragmentaire et « cercle vicieuX »
}

Les manuscrits de De l'essence double du langage de Ferdinand de Saussure

\section{Kazuhiro Matsuzawa}

\section{(2) OpenEdition} Journals

Édition électronique

URL : http://journals.openedition.org/genesis/1037

DOI : $10.4000 /$ genesis. 1037

ISSN : 2268-1590

Éditeur :

Presses universitaires de Paris Sorbonne (PUPS), Société internationale de génétique artistique littéraire et scientifique (SIGALES)

Édition imprimée

Date de publication : 15 novembre 2012

Pagination : 41-58

ISBN : 978-2-84050-869-4

ISSN : 1167-5101

Référence électronique

Kazuhiro Matsuzawa, « Puissance de l'écriture fragmentaire et « cercle vicieux » », Genesis [En ligne], 35 | 2012, mis en ligne le 15 novembre 2014, consulté le 19 juin 2020. URL : http://

journals.openedition.org/genesis/1037 ; DOI : https://doi.org/10.4000/genesis.1037 


\title{
Puissance de l'écriture fragmentaire et « cercle vicieux 》 Les manuscrits de De l'essence double du langage de Ferdinand de Saussure
}

\author{
Kazuhiro Matsuzawa
}

$\mathrm{D}$ epuis la découverte inattendue, en 1996, dans l'orangerie de l'hôtel genevois de la famille de Saussure, de notes encore inédites du grand maître, l'étude des manuscrits connaît un certain regain d'intérêt. Une partie considérable de ces manuscrits ont été retrouvés dans une grande enveloppe sur laquelle Saussure avait noté « Science du langage »; ils se trouvent actuellement conservés à la Bibliothèque de Genève sous la cote «Arch. de Saussure 3721 ». Les manuscrits sont paginés de 1 à 274 par la Bibliothèque de Genève selon l'ordre choisi par Rudolf Engler. Parmi eux un grand nombre de feuillets, contenus dans de petites enveloppes portant l'inscription « De l'essence double du langage » ou " De l'essence etc » de la main de Saussure, ont été détachés par Engler qui a subdivisé l'ensemble en vingt-neuf petits chapitres, dont certains sont encore subdivisés par des lettres. Ils sont publiés sous le titre « De l'essence double du langage » dans les Écrits de linguistique générale ${ }^{2}$, avec les autres manuscrits répartis dans les trois autres sections : "Item et Aphorismes », "Autres écrits de linguistique générale », et « Notes préparatoires pour le cours de linguistique générale ». Cette édition contient non seulement le dossier "Science du langage », mais aussi les autres notes autographes de Saussure déjà publiées par Engler 3 .

Devant la masse des manuscrits de « De l'essence double du langage », l'impression qui prédomine est celle d'être face à des fragments inachevés apparemment en désordre. De l'état éparpillé des manuscrits faut-il conclure qu'ils ne sont que des brouillons dépourvus de toute cohérence qui ne méritent pas un examen critique ? Par ailleurs la publication des Écrits de linguistique générale ne cesse d'attirer l'attention, par exemple, sur l'esquisse de certains concepts qu'on ne retrouve pas ailleurs. S'agirait-il alors, comme le disent les éditeurs des $E L G$, d' « esquisses consistantes d'un livre sur la linguistique générale » $(E L G$, p. 12) que Saussure aurait rédigé à côté des textes fragmentaires ? Dans cette perspective, la pensée linguistique de Saussure aurait besoin d'être développée au-delà de l'état d'ébauche où il l'a laissée. Si tant est que les notes étaient une esquisse théorique à développer sous forme de linguistique générale, on se demandera pourquoi Saussure a renoncé au projet d'un tel livre

1. Selon le catalogue de la Bibliothèque de Genève. Voir aussi Ferdinand de Saussure, Wissenschaft der Sprache: Neue Texte aus dem Nachlaß, éd. Ludwig Jäger, Elisabeth Birk und Mareike Buss, Frankfurt am Main, Suhrkamp Verlag, coll. «Suhrkamp Taschenbuch Wissenschaft», 2003, p. 19.

2. Édition établie et éditée par Simon Bouquet et Rudolf Engler, Paris, Gallimard, 2002. Nous désignerons désormais les Écrits de linguistique générale (éd. Bouquet, Engler) par ELG.

3. Édition critique du "Cours de linguistique générale » de Ferdinand de Saussure, Wiesbaden, Otto Harrassowitz, 1967-1974.

\author{
Les manuscrits de \\ De l'essence double \\ du langage
}


qu'avec son génie il aurait rédigé sans trop de difficultés. En prenant implicitement le point de vue rétrospectif qui assigne à Saussure la fonction du père du structuralisme, ne court-on pas le risque d'être encore prisonnier du succès fulgurant du Cours de linguistique générale, rédigé et publié par deux disciples trois ans après la mort du maître ? Dans cette emprise, on se condamne, en effet, à méconnaître la question de l'écriture fragmentaire qui caractérise d'une façon si saillante les dossiers manuscrits de Saussure. Les notes découvertes en 1996 ne sont-elles pas en effet aussi fragmentaires que les manuscrits connus jusqu'à ce jour ? Comment ne pas reconnaître à ce type d'écriture une certaine importance et comment ne pas s'y arrêter?

Or toute tentative d'édition critique des manuscrits oscille entre deux tendances contraires : visibilité et lisibilité, transcription et réécriture. Ainsi elle peut revêtir diverses formes qui évoluent entre deux types extrêmes : l'édition diplomatique, qui respecte la disposition propre à chaque feuille écrite, et l'édition dite standard qui ne conserve que le texte final sous une forme très lisible. Chaque édition devrait être évaluée à sa juste valeur par rapport à l'usage qu'on en préconise et au public auquel elle s'adresse. L'édition de Gallimard, relevant du second type, s'adresse au public cultivé en rendant les manuscrits directement lisibles. Mais du point de vue de la critique génétique, elle est peu satisfaisante : les problèmes d'arrangement et de transcription mis à part, les manuscrits sont nettoyés de leurs repentirs nombreux et complexes, perdant de ce fait la richesse de l'écriture en train de penser. Elle ne rend pas compte de l'ensemble du dossier de la «Science du langage 4 » et de son état, nécessaires à l'appréciation des chercheurs avertis. Face à cette publication, à laquelle nous renverrons cependant, nous avons dû revenir aux manuscrits eux-mêmes pour aborder de front la question de l'écriture fragmentaire de Saussure.

Force est de constater que la fragmentarité caractéristique des manuscrits de Saussure a toujours été signalée plutôt qu'explorée et ne fait que rarement l'objet d'une critique approfondie. Quelques exceptions sont cependant à rappeler. Ainsi, pour T. De Mauro, la pensée de Saussure n'apparaît pas comme un ensemble de dogmes mais comme la patiente exploration de liaisons entre de multiples «points de vue ». Il signale un intéressant parallélisme avec les difficultés rencontrées par Wittgenstein sur le problème de la présentation ${ }^{5}$. À la suite de F. Gadet, C. Bota reconnaît une importance actuelle à la question de l'ordre de présentation 6 . Selon L. Jäger, ce qui conduit Saussure à un style aphoristique dans ses ébauches est la conscience de l'impossibilité d'accomplir l'énorme tâche que représente une réforme radicale de la linguistique ${ }^{7}$. On ne peut que s'accorder avec Jäger pour dire que l'attitude épistémologique de Saussure n'a trouvé son expression

4. Ainsi les pages 202-215, 217-237, 255-274 sous la même cote ne sont ni transcrites ni mentionnées dans ELG. 5. Ferdinand de Saussure, Cours de linguistique générale, éd. T. De Mauro, Paris, Payot, 1972, p. 408-409.

6. Françoise Gadet, Saussure. Une science de la langue, Paris, Presses universitaires de France, 1997, p. $26-27$; Cristian Bota, « La question de l'ordre dans les cours et les écrits saussuriens de linguistique générale. Essai de refonte géométrique », Cahiers Ferdinand de Saussure, n 55, 2002. Voir aussi Kazuhiro Matsuzawa, « Notes pour un livre sur la linguistique générale », dans S. Bouquet (dir.), Saussure, Paris, Éditions de 1'Herne, coll. « Cahiers de l'Herne, nº $76 », 2003$.

7. Ludwig Jäger, «La pensée épistémologique de F. de Saussure », dans Saussure, Paris, Éditions de l’Herne, op. cit., p. 204. 
adéquate que dans des formes aphoristiques. Or le renoncement à la construction d'un édifice théorique a en lui-même une dimension théorique qui mérite encore notre réflexion critique. Au lieu de thématiser, ainsi que Jäger le fait d'ailleurs très judicieusement, le problème des conditions du jugement d'identité, on se propose de montrer dans ce qui suit que l'attention portée à l'écriture fragmentaire de Saussure invite à mettre en évidence les transformations par lesquelles cette écriture ouvre un nouvel espace de réflexion en mettant en question la prétention d'une pensée linéaire attachée à construire l'édifice théorique de la linguistique générale.

On doit l'ordre de la présentation des manuscrits de « Science du langage » sous le titre «De l'essence double du langage » au travail laborieux de R. Engler. La première note de cet ensemble s'ouvre dans la présentation d'Engler sous le titre « 1 Préface », qui occupe le centre de la page. Le lecteur des ELG aura l'impression d'assister au véritable commencement d'une réflexion et comprendra bien la raison qui conduit Engler à placer cette note au début de « De l'essence double du langage ». Vu l'importance de cette note, on la citera in extenso (voir fig. 1 la reproduction de ce manuscrit et sa transcription 8 ).

On retrouve dans le dossier de Science du langage quelques indices du projet d'un livre indiqué par les mentions « cet opuscule » $(71 ; E L G, 9$, p. 45), « La division de ce livre » (195;ELG, p. 95). S'agit-il alors du manuscrit de la préface ? À consulter le manuscrit, on s'aperçoit sans peine qu'un trait oblique indique que «Préface » a été ajouté après coup par la main de Saussure dans la marge gauche en haut de la page. Cependant l'examen des manuscrits nous apprend que les titres sont écrits dans la plupart des cas en haut et au centre du feuillet. Par ailleurs, cette page reste dans un état de brouillon comportant un certain nombre d'ajouts et de ratures. On peut en effet trouver la mention « avant-propos » (157 ; $E L G, 27$, p. 76) qui indique un autre commencement possible. Contrairement à ce qu'en font les éditeurs des $E L G$, l'ajout marginal de « Préface » indique le caractère provisoire et précaire du brouillon. Dès le début des $E L G$, on assiste ainsi à une sorte d'altération - à tout le moins une interprétation - qui transforme l'inachevé ou le provisoire en définitif ou arrêté.

8. Le code de transcription dans le présent article est le suivant : l'ajout interlinéaire ou marginal dans le manuscrit est indiqué entre soufflets $(<\ldots\rangle)$ et dans un corps plus petit ; les mots raturés ou soulignés et les majuscules sont reproduits tels quels dans la transcription; les abréviations sont développées. « [...]» indique qu'il s'agit d'une lecture conjecturale ou de l'intervention de l'éditeur. On a profité de «Ferdinand de Saussure. De l'essence double du langage, transcription diplomatique établie par Rudolf Engler d'après le manuscrit déposé à la Bibliothèque de Genève (1996) (voir revue Texto !, décembre 2004-juin 2005 [en ligne], <www.revue-texto.net/Saussure/De_ Saussure/Essence/Engler.html>), qui a servi de base à l'édition de Gallimard. Notre transcription est cependant parfois différente de celle de R. Engler.

\section{L'absence de point de départ}




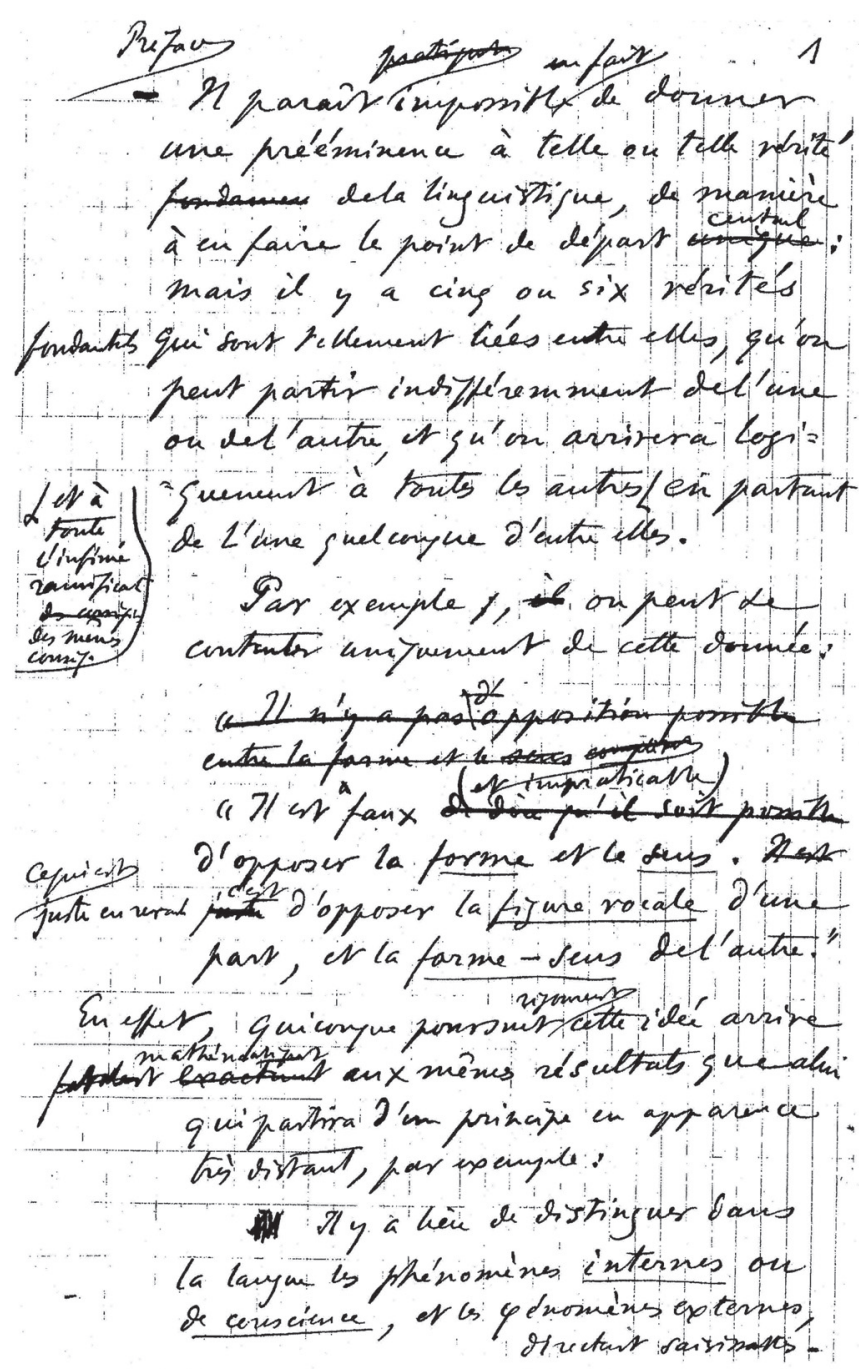

Fig. 1 : «De l'essence double du langage » (Bibliothèque de Genève, Arch. de Saussure 372, p. 1)

$<$ Préface $>$

Il paraît <pratiquement $>$ impossible <en fait $>$ de donner une prééminence à telle ou telle vérité fondamen de la linguistique, de manière à en faire le point de départ unique <central $>$ : mais il y a cinq ou six vérités $<$ fondamentales $>$ qui sont tellement liées entre elles, qu'on peut partir indifféremment de l'une ou de l'autre, et qu'on arrivera logiquement à toutes les autres <et à toute l'infinie ramification des mêmes conséqs $>$ en partant de l'une quelconque d'entre elles.

Par exemple, it on peut se contenter uniquement de cette donnée :

*Ill n'y a pas < d'> opposition possible entre la forme et le sens.

«Il est faux de dire qu'il soit possible $<$ (et <eomplèt> impraticable) $>$ d'opposer la forme et le sens. Hl est juste $<$ Ce qui est juste en revanche, c'est $>$ d'opposer la figure vocale d'une part, et la forme-sens de l'autre. »

En effet, quiconque poursuit <rigoureusement $>$ cette idée arrive exactement < fatalement $>$ mathématiquement $>$ aux mêmes résultats que celui qui partira d'un principe en apparence très distant, par exemple :

HI Il y a lieu de distinguer dans la langue les phénomènes internes ou de conscience et les phénomènes externes, directement saisissables $(1 ; E L G, 1, \text { p. } 17)^{9}$.

9. Pour la citation du manuscrit de De l'essence double du langage de Saussure, on a indiqué la pagination donnée par la Bibliothèque de Genève, le chapitre et la page des $E L G$. Pour les autres manuscrits, on a indiqué seulement la pagination de la Bibliothèque de Genève et celle des $E L G$. Ainsi « $1 ; E L G, 1$, p. 17 » signifie $\mathrm{f}^{\circ} 1$ de la Bibliothèque de Genève, chapitre 1, p. 17 des $E L G$. Pour les autres documents de la Science $d u$ langage, on a indiqué la pagination de la Bibliothèque de Genève et la page des ELG. Pour les anciens documents, on a indiqué seulement la page des $E L G$. 
Continuons d'observer ce feuillet. Le texte s'articule en deux parties aisément identifiables. Après l'exposé de la thèse générale qui affirme d'emblée l'absence de "point de départ central », vient un exemple («Par exemple ») supposé en fournir l'illustration.

Le premier paragraphe est doublement déterminant. En général, les considérations de départ, sous peine de rester lettre morte, supposent les développements ultérieurs auxquels elles sont censées servir d'entrée en matière. Le problème que soulève la thèse initiale de l'absence de départ est en premier lieu qu'il n'est plus possible de concéder une quelconque « prééminence à telle ou telle vérité ». D'où peut-on partir? Au commencement, Saussure se trouve donc confronté à la difficulté redoutable qui consiste à devoir s'assigner un point de départ tout en affirmant que celui-ci est illusoire. Le paradoxe n'est nulle part aussi éclatant qu'ici. Le commencement se perçoit comme déjà emporté dans quelque chose qui le rend à la fois impossible et obligé. On remarquera la substitution de « central » à « unique », qui va dans le sens de l'atténuation. Replacée dans le contexte, elle renforce au contraire l'absence de point de départ : même le point de départ, du moins central, sinon unique, n'est pas possible.

En second lieu, la thèse initiale, obligeant la science du langage à renoncer au progrès linéaire qui conduit du début du système à son terme, signale à celle-ci le besoin de se constituer sous la forme de renvois circulaires. T. De Mauro fait remarquer les similitudes de ce passage avec le premier paragraphe de l'Encyclopédie des sciences philosophiques de Hegel ${ }^{10}$. Le philosophe allemand écrit en effet d'une façon qui rappelle Saussure : "Mais la difficulté d'instituer un commencement se présente par là en même temps, puisqu'un commencement, en tant qu'il est un immédiat, institue une présupposition ou bien plutôt en est lui-même une ${ }^{11}$. » Hegel affirme que le commencement présuppose quelque chose qu'on ne peut pas démontrer. Or, dans un travail voué à se présenter progressivement, il arrive souvent qu'on se contente d'une définition globale susceptible de développements ultérieurs. Dans ce cas, il suffit d'attendre l'instant de la spécification. Mais chez Saussure comme chez Hegel, le problème du commencement est de nature différente, car il s'agit d'une implication mutuelle et complexe du conditionnant et du conditionné, du déterminant et du déterminé, d'où l'absence de point de départ ou de principe premier. Une pareille affirmation se retrouve dans le dossier découvert en 1996, intitulé par Bouquet et Engler « Nouveaux Item » : «Il est $<$ profondément $>$ faux de s'imaginer qu'on puisse faire une synthèse radieuse de la langue, en partant d'un principe < déterminé> qui se développe et s'incorpore avec [tout le reste]. Il [ce livre] montre qu'on ne peut comprendre ce qu'est la langue qu'à l'aide de quatre ou cinq principes sans cesse intercroisés d'une manière qui semble faite exprès pour tromper les plus habiles et les plus attentifs à leur propre pensée » $(195: E L G$, p. 95$)$; « Ne parlons ni d'axiomes, ni de principes, ni de thèses » (ELG, p. 123).

Sous la plume de Saussure revient à plusieurs reprises l'idée que la science du langage ne se constitue pas sous la forme d'un système théorique dans lequel, à partir d'un seul et unique principe, serait déduit tout le reste.

10. Ferdinand de Saussure, Scritti inediti di linguistica generale, traduit et édité par Tullio De Mauro, Roma/Bari, Laterza, 2005, p. 6.

11. G.W.F. Hegel, Encyclopédie des sciences philosophiques, Paris, Vrin, 1979, p. 164. 


\section{La figure vocale/la « forme-sens » et les phénomènes externes/ internes}

Il convient d'examiner à présent l'exemple que Saussure donne ensuite. Saussure remet en question la figure vocale comme point de départ sûr. Au dernier tiers du XIXe siècle, sous l'influence de l'école des néogrammairiens, on est habitué à penser que «Prendre la langue par le côté du phénomène vocal est certainement la manière la plus simple de toutes de l'aborder » (38:ELG, 5b(3)(4), p. 33). Saussure critique cette conception dominante qui pense que la figure vocale, étant un objet sensible, indéniable, fournit une base empirique à la linguistique : il défend la thèse selon laquelle c'est l'indissolubilité de la forme et du sens qui fait naître un signe linguistique. Il faut faire attention ici à un certain flou terminologique à l'égard du concept de signe. Dans « De l'essence double du langage », Saussure emploie le terme « signe » au sens qu'il donnera postérieurement au terme d' " image acoustique » ou de « signifiant ». La suite des notes reprend sans cesse, avec une certaine ténacité, ce lien singulier du son et du sens dans le domaine linguistique. Ainsi, l'ensemble des feuillets 2a fait remarquer qu'une identité linguistique implique «l'association de deux éléments hétérogènes » $(2 ; E L G, 2 \mathrm{a}(1), \mathrm{p} .18)$. Or, selon Saussure, si on poursuit rigoureusement cette idée, on arrivera aux mêmes résultats que celui qui partira d'une autre idée concernant la distinction entre les «phénomènes internes ou de conscience et les phénomènes externes, directement saisissables ». Aucun commentaire n'est donné à l'égard du rapport entre les deux idées. Mais Saussure insiste sur « de conscience " afin d'affirmer que l'objet propre de la linguistique relève de phénomènes internes. La distinction entre la «forme-sens » et la figure vocale est transposable à celle entre les phénomènes internes et les phénomènes externes. En tant que réalité physique, le langage est directement saisissable, soit dans le temps s'il est parlé, soit dans l'espace s'il est écrit. Mais dans les deux cas, ce ne sont que des phénomènes externes. Or l'objet principal de la linguistique relève d'un " premier domaine <intérieur>, psychique, où existe le signe autant que la signification, l'un indissolublement lié à l'autre » $(11 ; E L G, 2$ d, p. 21), car « le mot pas plus que son sens n'existe hors de la conscience que nous en avons » $(172 ; E L G, 29 b(1)$, p. 83$)$.

La distinction de trois domaines que propose le feuillet 8 est très éclairante à cet égard. Sur un manuscrit très travaillé $(69,70 ; E L G, 8(1)$ et $8(2)$, p. 43-44), nous pouvons extraire :

I. Domaine non linguistique de la pensée pure.

II. Domaine linguistique du signe vocal (Sémiologie) qui est à la fois celui de la pensée relative, de la figure vocale relative, et de la relation entre ces deux.

III. Domaine linguistique du son pur.

Saussure se garde d'une double réduction du langage à la pensée pure et à la figure vocale. Après l'école de la grammaire comparée fondée par F. Bopp, qui «n'a vu l'idiome qu'à travers le voile de l'écriture » $(198 ; E L G$, p. 130), vient l'école des néogrammairiens. Tout en reconnaissant l'importance du sujet parlant, cette école, toute à sa volonté de se constituer en science positive, s'attache à subordonner autant que possible l'élément immatériel à l'élément matériel et proprement physique du langage qu'est la figure vocale. La délimitation saussurienne de l'objet de la linguistique comme phénomène interne de la conscience définit un cadre de connaissance linguistique et implique des règles de traitement du nouvel objet ou de l'objet renouvelé, nommé enfin le langage. 
Mais si l'essence de la « forme-sens » est psychique, spirituelle, immatérielle, comment peut s'opérer cette transmutation qui fait d'emblée de la pensée et de la figure vocale une telle entité ? Saussure a quelque peine à dire ce que peut être l'association incorporelle de la « formesens ». La réflexion préliminaire sur la nature de l'objet du langage, en particulier sur la formesens, ne dispose d'aucun moyen de rendre immatérielle la figure vocale. Une question qui se pose alors inévitablement est celle-ci : le concept du signe linguistique comme «forme-sens » a-t-il été vraiment déduit de l'observation de la nature de l'objet linguistique ?

Le lieu d'où Saussure parle de l'immatérialité s'éclaire mieux dans le passage suivant (fig. 2) :

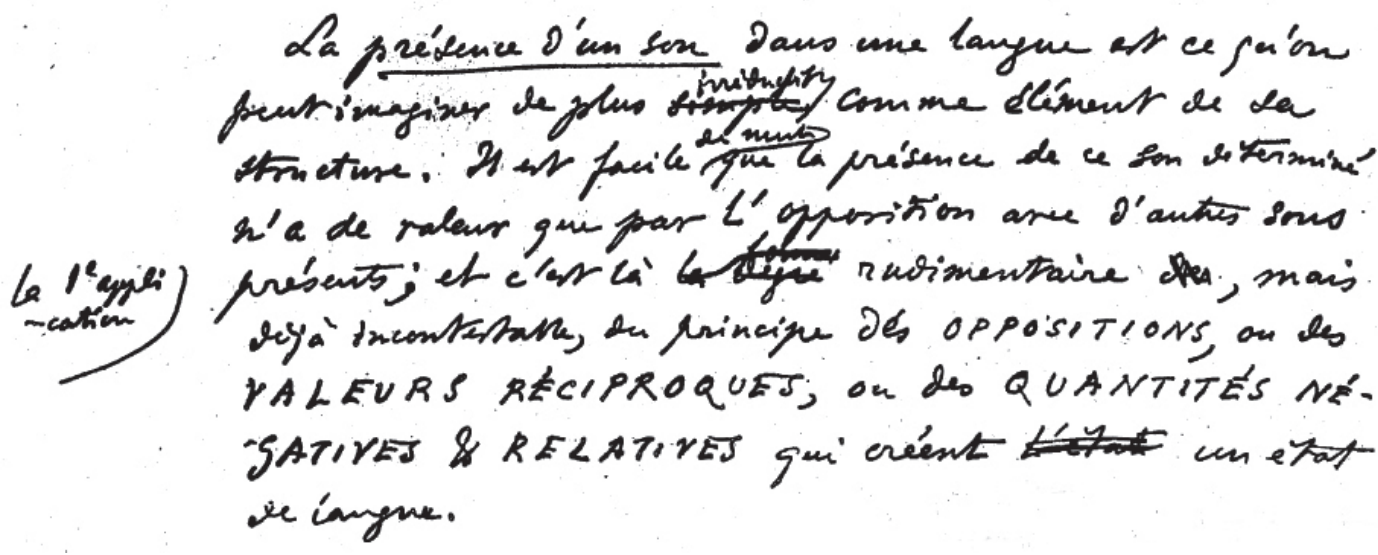

Fig. 2

La présence d'un son dans une langue est ce qu'on peut imaginer de plus simple <irréductible> comme élément de sa structure. Il est facile $<$ de montrer $>$ que la présence de ce son déterminé n'a de valeur que par l'opposition avec d'autres sons présents; et c'est là te degré $<$ ta forme $><$ la $1^{\mathrm{e}}$ application $>$ rudimentaire des, mais déjà incontestable, du principe des OPPOSITIONS, ou des VALEURS RÉCIPROQUES, ou des QUANTITÉS NÉGATIVES \& RELATIVES qui créent L'état un état de langue $(23 ; E L G, 3 \mathrm{c}$, p. 25).

Ce qu'il importe de remarquer ici, c'est que Saussure explique la détermination relative du son par la première application rudimentaire du principe des oppositions ou des valeurs réciproques, principe qui n'est pas encore présenté comme tel avant la question de la formesens. La première phrase de ce passage fait à plusieurs reprises l'objet d'un développement dans la suite du même feuillet : «La présence d'une corrélation <ressentie> entre deux sons (restant encore dénuée du reste de toute signification proprement dite) », ensuite « La présence d'une corrélation apparente < ressentie> entre deux sons à laquelle commence à se joindre une différence de [signification est un troisième degré] ». L'identité de chaque signe comme forme-sens apparaît ainsi comme déterminée par des rapports de différence entre eux sur le plan de la signification autant que sur le plan du son. De même, « Une forme est une figure voeale qu' $<$ Une figure vocale devient une forme depuis l'instant crucial où $>$ on l'introduit dans le jeu de signes appelé langue » (53:ELG, f. 6e(3), p. 38). La substitution du verbe « devenir » au verbe « être » dans cette réécriture met en relief une transformation capitale par laquelle la figure vocale se trouve désubstantialisée. Ce « jeu de signes appelé langue » 
est le fonctionnement d'un système de valeurs différentielles où est à l'œuvre ce « principe fondamental de la sémiologie »: «Il n’y a dans la langue ni signes, ni significations, mais des DIFFÉRENCES de signes et des DIFFÉRENCES de signification » $(144 ; E L G$, $22 b(1), p .70)$ et ces différences de nature différente «n'arrivent jamais à se correspondre directement ». Le feuillet 8(1) précise la troisième relation selon laquelle le signe linguistique comme forme-sens « est à la fois celui de la pensée relative, de la figure vocale relative, et de la relation entre ces deux » $(69 ; E L G, 8(1)$, p. 44). « La relation entre ces deux » désigne le découpage simultanément opéré par la langue sur le plan des significations et celui des sons. L'immatérialité de la « forme-sens » est donc un des effets de ce «principe fondamental de la sémiologie » où tout est négatif et différentiel. Au lieu de partir du premier principe, Saussure remonte, à travers l'analyse de l'immatérialité de la «forme-sens », à ce que celle-ci présuppose. La réflexion préliminaire sur la nature du signe linguistique s'inscrit donc déjà dans la dépendance du principe de la négativité qui, lui, révèle son fonds de présuppositions. Le «principe fondamental » de la sémiologie contient l'amorce de son émergence dans ses effets. Ce mouvement dans lequel Saussure est emporté à son insu, le philosophe allemand de Etre et Temps l'appellera cercle herméneutique.

La preuve scientifique ne doit pas présupposer ce qu'elle a pour tâche de fonder. Mais l'explication doit déjà se mouvoir à chaque fois dans ce qui est entendu et s'en nourrir, comment peut-elle alors faire venir à maturation des résultats scientifiques sans se mouvoir dans un cercle ${ }^{12}$ ?

Pris dans le cercle herméneutique, Saussure ne cesse d'en appeler au «principe fondamental » tout en le présupposant. On remonte au présupposé implicite au fur et à mesure qu'on avance, car on ne peut prendre le point de départ que dans ce qui est déjà constitué.

\section{Phonétique et morphologie}

Dans « De l'essence double du langage », la troisième dualité qui succède aux dualités précédemment envisagées est celle de la phonétique et de la morphologie. Au dernier tiers du XIX ${ }^{e}$ siècle, la morphologie est définie comme l'étude des formes du langage, tandis que la phonétique est l'étude des sons du langage. Comment Saussure arrive-t-il à remettre en question cette distinction apparemment naturelle pour y voir la manifestation de la dualité foncière du langage ? Il part, semble-t-il, du fait inaperçu et banal de l'alternance (fig. 3) :

12. M. Heidegger, Être et Temps, Paris, Gallimard, 1986, p. 198. 


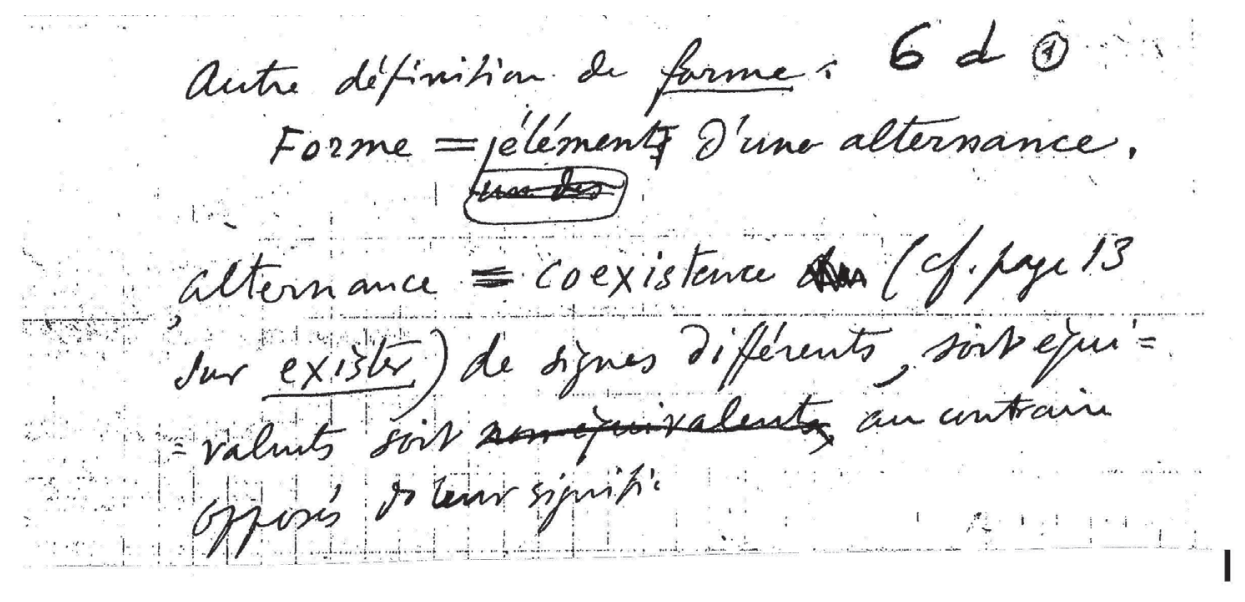

Fig. 3

Autre définition de forme:

Forme $=<$ tnd des $>$ éléments d'une alternance.

Alternance $=$ coexistence des (cf. page 13 sur exister) de signes différents, soit équivalents soit non équivalents, au contraire opposés dans leur signific[ation] (48;ELG, 6d(1), p. 36).

$$
\begin{aligned}
& \text { (1) rovipas phoneticus. } 5 \text { a (1) } \\
& \text { Les alternanus sont los sifférences } \\
& \text { vocales (") existont au même monent } \\
& \text { entwo farmes gue l'on juge rejurésenter, } \\
& \text { ite ì ith fulcorym, une unit' } \\
& \text { mophotogine - Phis ou mor'us large, } \\
& \text { mais à dencel'exclurion de l'unite' denmiezo } \\
& \text { su'st L'identite moprtologine. }
\end{aligned}
$$

Fig. 4

$<(1)$ non pas phonétiques.>

Les alternances sont les différences vocales(1) existant au même moment entre formes que l'on juge représenter, the unité à un titre quelconque, une unité morphologique - plus ou moins large, à la seule <mais à l'> exclusion de l'unité dernière qui est l'identité morphologique (34;ELG, 5a(1), p. 30, fig. 4). 
L'ensemble des phénomènes que recouvre la notion d'alternance s'explique par la « coexistence », « au même moment », de signes différents que l'on juge représenter une unité morphologique. On notera ici une substitution intéressante, " opposés » à " non équivalents », ce qui suggère une intervention subreptice du système de différences. Saussure considère les alternances « comme parfaitement <éminemment> morphologiques <puisqu'elles sont instantanées> 》 $(123 ; E L G$, f. 19(2), p. 63). Les alternances relèvent de la morphologie qui suppose un ordre grammatical et stable de la langue à un moment donné de l'histoire. La phonétique, au contraire, s'occupe du changement historique du son qui échappe à la conscience des sujets parlants. Saussure voit une différence radicale entre les deux points de vue que supposent ces deux études. L'essentiel est que la linguistique synchronique s'occupe des phénomènes internes du langage dont le sujet parlant a toujours la conscience à quelque degré que ce soit. Ainsi la distinction entre la phonétique et la morphologie doit être repensée et reformulée par rapport à la conscience des sujets parlants. On s'aperçoit rétrospectivement que c'est la conscience des sujets parlants à un moment donné qui préside à la distinction de la forme-sens et de la figure vocale, comme à la distinction des phénomènes internes et externes, en même temps qu'elle est le fruit d'un approfondissement.

Sans entrer dans le détail, on se bornera à entrevoir ici une remise en question de la distinction de la morphologie et de la phonétique. Saussure considère "la prétendue règle phonétique dressée en se renfermant dans un état donné de langue » $(116 ; E L G$, f. 15(7), p. 59) comme une règle morphologique. En prenant l'exemple de la prétendue règle phonétique du sandhi sanskrit, selon laquelle « $\mathrm{s}$ après $\mathrm{k}$, $\mathrm{r}$, et les voyelles autres que [ā /ă] devient ș », Saussure n'y reconnaît pas une modification phonétique, mais considère que « Sa règle est < finalement> l'expression d'une alternance, fait essentiellement morphologique »(115;ELG, f. 15(7), p. 59). L'alternance, tout comme l'analogie, suppose l'ordre de la coexistence des termes en question, coexistence dont le sujet parlant a la conscience. Saussure tente ainsi d'élargir l'objet synchronique que l'alternance ou la morphologie peut recouvrir.

Quant à la figure vocale, elle ne se transforme en forme que dans le rapport de différence avec d'autres formes, mais cette différence n'est à l'œuvre que dans les sujets parlants. 


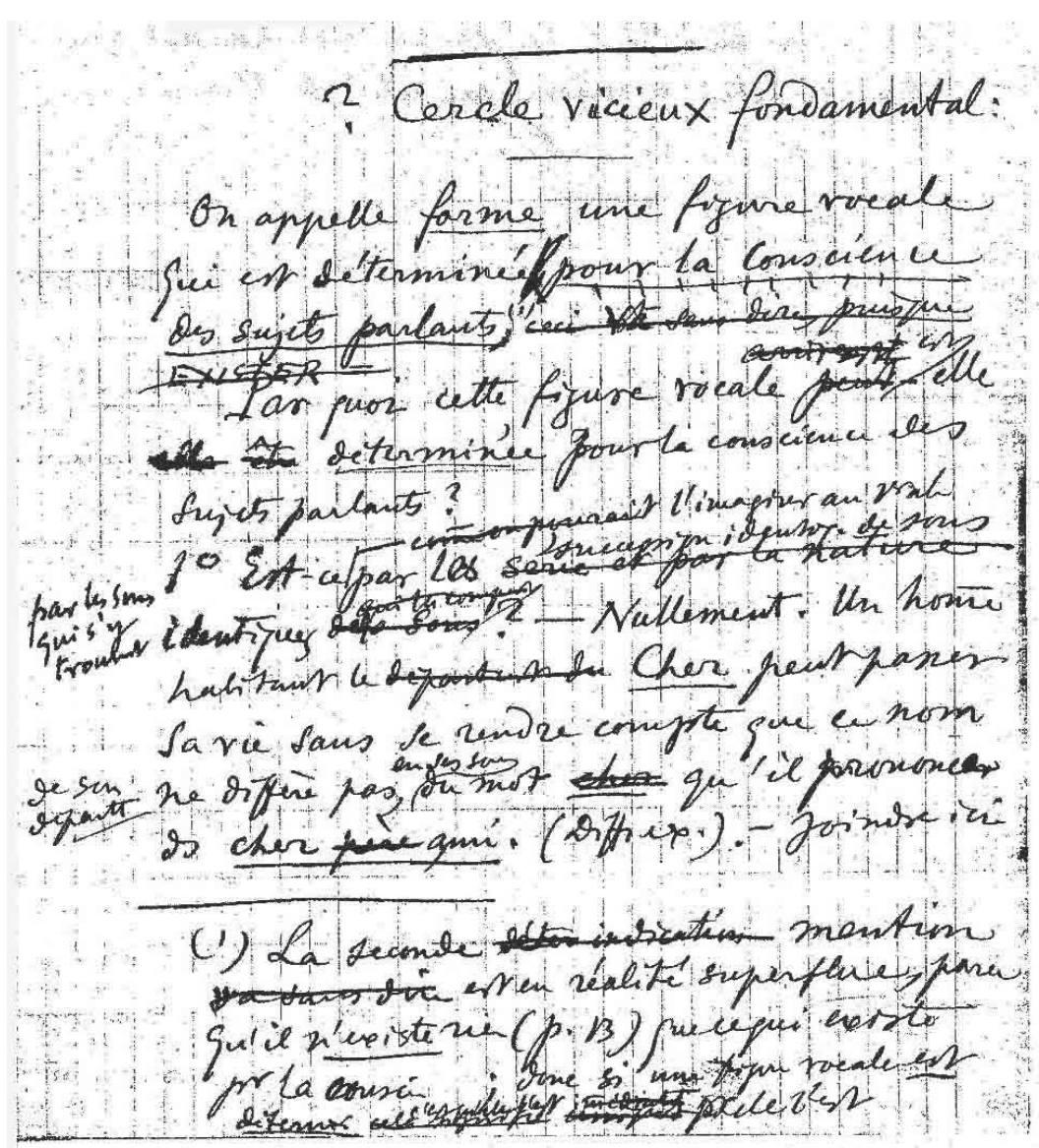

Fig. 5 : «De l'essence double du langage » (Bibliothèque de Genève, Arch. de Saussure 372)

? Cercle vicieux fondamental :

On appelle forme une figure vocale qui est déterminée pour la conscience des sujets parlants. ${ }^{(1)}$ $[\ldots]$

(1) La seconde déter <indieation><mention> en réalité superflue, parce qu'il n'existe rien (p. 13) que ce qui existe pr la conscience; donc si une figure vocale est déterminée, eeta signiffe uniquement <c'est qu'elle l'est immédiatement> qu'elle l'est (81;ELG, f. 10a, p. 49).

C'est au moment où est interrogé le rapport entre la forme, la différence et la conscience des sujets parlants que la question «? Cercle vicieux fondamental » dévoile ses enjeux. La forme suppose la conscience des sujets parlants en dehors de laquelle la détermination par la différence n'est pas possible. Le principe sémiologique de la différence négative suppose un état statique et morphologique fondé sur la conscience des sujets parlants. En dehors de celle-ci, un tel principe ferait de la langue un être ayant une existence propre, indépendante de l'esprit humain, ce qui céderait à « l'illusion des êtres linguistiques menant une existence indépendante » $(18 ; E L G, 3 \mathrm{~b}(2)$, p. 23). Il s'agit de la conception organiciste du langage, critiquée déjà avec virulence par Whitney et l'école des néogrammairiens avant Saussure. Mais la conscience des sujets parlants est en retour toujours affectée de la négativité différentielle sans laquelle elle n'arrivera pas à distinguer les phénomènes langagiers. Il y a donc une interaction et une interdépendance entre la conscience des sujets parlants et le principe de la sémiologie, qui s'impliquent mutuellement. 


\section{Le cercle vicieux}

Voici un passage qui laisse deviner l'hésitation de Saussure causée par le problème de la réversibilité de l'ordre (fig. 6) :

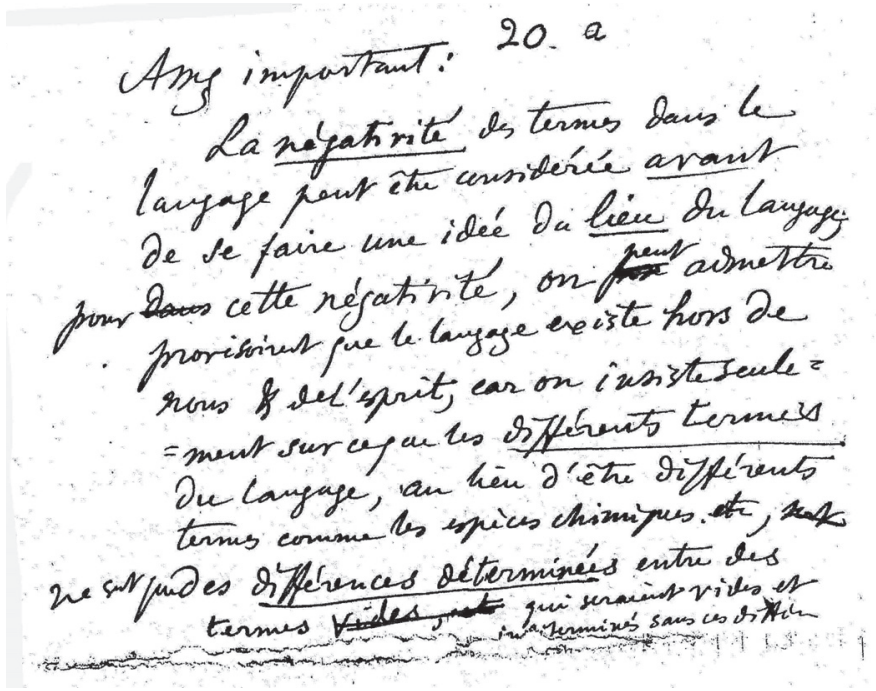

Fig. 6

Assez important :

La négativité des termes dans le langage peut être considérée avant de se faire une idée du lieu du langage ; dans < pour> cette négativité, on pose < peut> admettre provisoirement que le langage existe hors de nous \& de l'esprit, car on insiste seulement sur ce que les différents termes du langage, au lieu d'être différents termes comme les espèces chimiques, etc., sont $<$ ne sont que $>$ des différences déterminées entre des termes vides, et qui seraient vides et indéterminés sans ces différences $(133 ; E L G$, f. 20a, p. 64).

Comme l'indique l'expression « on <peut> admettre provisoirement », Saussure envisage ici la possibilité d'un autre ordre d'argumentation qui consisterait à présenter les différents termes comme des différences déterminées avant de montrer « le lieu du mot, la sphère où il se réalise <acquiert une réalité> qui « est purement l'esprit <ESPRIT> atsssi qui est aussi le seul $\underline{\text { lieu }}$ de <où il ait> son sens » $(174 ; E L G$, f. $29 \mathrm{~b}(4)$, p. 83$)$. Notons au passage que Saussure insiste sur l'esprit comme lieu du mot. Or, selon ce nouvel ordre, le principe de la différence viendra avant la nature immatérielle du langage. On peut figurer comme suit la réversibilité de l'ordre de l'exposé, telle qu'on l'a rencontrée jusqu'ici :

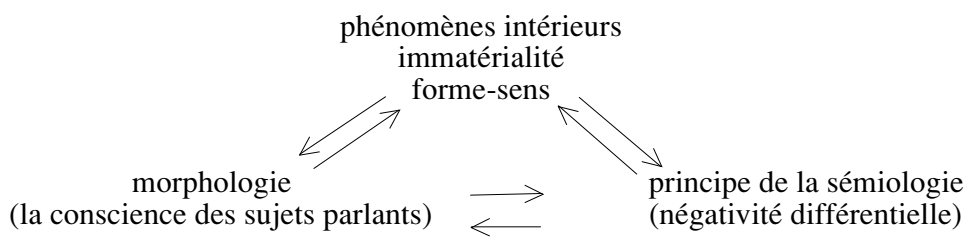


La hiérarchie s'estompe pour céder la place aux renvois circulaires. Le raisonnement de Saussure sous-entend l'interdépendance circulaire de ces « vérités ».

Cependant, si la lecture des manuscrits s'arrêtait à ce niveau de l'analyse, elle laisserait échapper l'importance de l'accent mis justement sur "l'essence double du langage ». Au moment même où le cercle a l'air de se boucler surgit une question d'un ordre différent. Saussure signale la nécessité d'une " séparation absolue entre l'être "langue" toujours momentané et le fait contingent que cet être "langue" est ordinairement destiné à se transmettre à travers le temps » $(93 ; E L G, 12(6)$, p. 55). À la lumière de la distinction des deux points de vue synchronique et diachronique, le schéma circulaire ci-dessus présuppose subrepticement l'abstraction faite de la dimension du temps de la transmission de la langue. Or, si tout état de langue est le résultat de la transmission, la dimension de ce temps, quoique invisible, n'est pas en dehors, mais s'avère inhérente à l'état de la langue qu'elle supporte. Une certaine stabilité qui assure un état de la langue est un effet du temps traditionnel, sans lequel toute convention, toute « forme-sens » ne serait pas possible pour la conscience des sujets parlants, dans la mesure où le langage n'est pas un simple instrument mais assimilé à la pensée même. Le principe de la sémiologie qui suppose les deux plans distincts du son et de la signification pour rendre compte de la relation entre les deux faces du signe n'est possible que sous « le regard impassible de Sirius 13 », car le sujet parlant immergé dans un état de la langue vit une intimité avec le langage sans s'attacher à une analyse aussi artificielle de celui-ci. C'est grâce au résultat de la linguistique diachronique qui éclaire sous un plein jour le déplacement entre le signifiant et le signifié que l'on peut rendre suffisamment compte de la relation interne du signe linguistique. En ce sens le principe de la négativité différentielle, pour être le principe de la langue, présuppose lui-même la dimension du temps traditionnel14. Si le temps apparaît ici rétroactivement comme condition de possibilité de la langue, c'est parce qu'il est toujours déjà présupposé par l'état de la langue.

Cependant, la dimension du temps ne constitue pas le fondement mais fait émerger une dualité fondamentale : le point de vue statique et le point de vue historique, qui s'impliquent mutuellement sans qu'on ne puisse accorder la priorité ni à l'un ni à l'autre. Ce qui serait originaire, ce n'est ni l'un ni l'autre, mais cette différence même entre les deux. Sous la plume de Saussure revient à plusieurs reprises l'expression « cercle vicieux » (fig. 7) :

13. E. Benveniste, Problèmes de linguistique générale, Paris, Gallimard, 1966, p. 51.

14. Sur ce point, voir Kazuhiro Matsuzawa, «Le "décousu" du troisième cours de linguistique générale et le cercle herméneutique », dans Le Projet de Ferdinand de Saussure, dir. J.-P. Bronckart, E. Bulea, C. Bota, Genève, Droz, 2010, p. 61-78. 


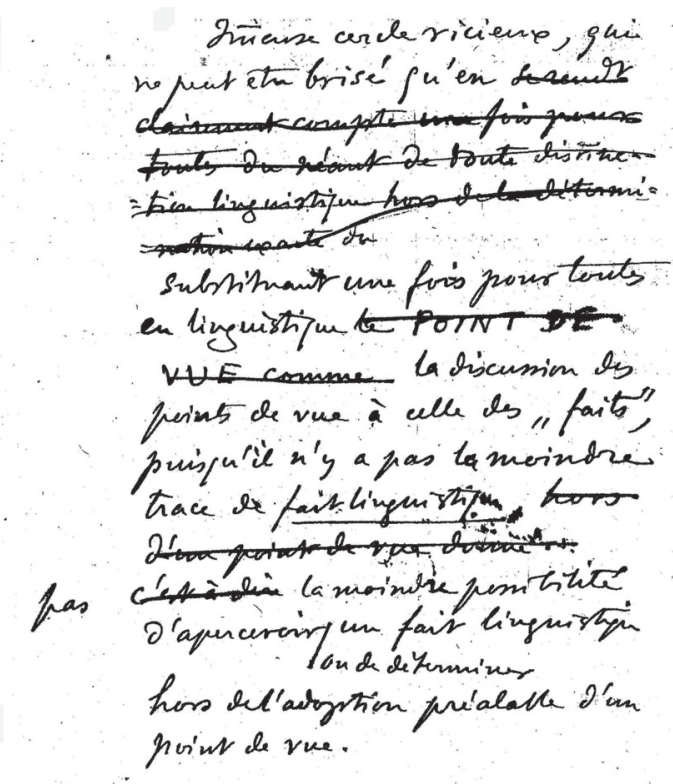

Fig. 7 : «De l'essence double du langage » (Bibliothèque de Genève, Arch. de Saussure 372)

Immense cercle vicieux, qui ne peut être brisé qu'en se rendant clairement eompte une fois pour toutes du néant de toute distinetion linguistique hors de la détermination exaete du substituant une fois pour toutes en linguistique te POINT DE VUE eomme la discussion des points de vue à celle des « faits », puisqu'il n'y a pas la moindre trace de fait linguistique, hors d'un point de vue donné e'est à dire <pas> la moindre possibilité d'apercevoir <ou de déterminer> un fait linguistique hors de l'adoption préalable d'un point de vue (21;ELG, f. 3b(5), p. 24).

Ce qui saute aux yeux, c'est l'expression assez directe du « néant de toute distinetion linguistique ». La prise de conscience du néant de toute existence linguistique déplace d'emblée l'intérêt du problème de la saisie d'un fait linguistique vers celui des points de vue. Mais la discussion des points de vue que Saussure substitue à celle des « faits » permet-elle d'échapper à cet immense cercle ? La classification de plusieurs points de vue ne semble pas constituer un ordre hiérarchique, mais un réseau dans lequel chaque point de vue renvoie l'un à l'autre en se chevauchant dans une présupposition mutuelle. Le 6 mai 1911, dans un entretien avec un de ses étudiants, Gautier, Saussure semble avoir reconnu l'idée d'une pensée circulaire :

Pour le moment, la linguistique générale m'apparaît comme un système de géométrie. On aboutit à des théorèmes qu'il faut démontrer. Or on constate que le théorème 12 est, sous une autre forme, le même que le théorème 33.

Première vérité : la langue est distincte de la parole. Ceci ne sert qu'à dépouiller le problème de tout ce qui est physiologique. Il ne reste ensuite qu'une matière purement psychique. Or il me semble qu'on vient à cette première nécessité par plusieurs voies opposées ${ }^{15}$. 
Saussure propose comme point de départ la distinction entre langue et parole pour en dégager le psychique, mais il ajoute tout de suite qu' " on vient à cette première nécessité par plusieurs voies opposées ». L'idée de renvoi circulaire trouve ici sa confirmation dans l'expression «par plusieurs voies opposées ». L'effort pour sortir du cercle vicieux fait invinciblement retomber dans le cercle, car le langage présente toujours une série de dualités dont chaque point de vue ne présente qu'une vue partiale. L'un des termes de la dualité apparaît comme l'ombre de l'autre. Le linguiste participe lui-même au jeu subtil de valeurs et de différences qui constitue l'objet-langue, et c'est en cela que le caractère problématique de l'opération du linguiste se révèle dans toute son ampleur : la valeur différentielle passe de l'objet à expliciter à l'opération même du linguiste pour qui distinguer c'est constituer : «C'est un trait de la langue comme de tout système sémiologique en général, qu'il ne puisse pas y avoir de différence chez elle entre ce qui distingue une chose et ce qui la constitue » (ELG, p. 123). La distinction métalinguistique se dote ainsi du pouvoir de constituer son objet. Une telle orientation « constructiviste » de la linguistique expose le linguiste au risque de l'arbitraire (au sens péjoratif du terme). Nous ne pouvons pas aborder cette question ici, à propos de laquelle nous nous bornons à signaler qu'elle hante Saussure qui s'engage dans un dédale de reflets éblouissants lorsqu'il écrit que "Nous sommes au contraire profondément convaincus que quiconque pose le pied sur le terrain de la langue peut se dire qu'il est abandonné par toutes les analogies du ciel et de la [terre] » (ELG, p. 220). Le cercle herméneutique ne conduit pas le grand maître à la découverte du principe premier, mais à une errance indéfinie dont témoignent ses manuscrits fragmentaires et lacunaires.

Dans le dossier manuscrit de 1996, on trouve une note où Saussure réfléchit sur son style aphoristique :

La division <de ce livre> en paragraphes minuscules a quelque chose d'un peu ridicule [...] C'est donc un terrain où chaque paragraphe doit rester comme un pieu solide enfoncé dans le marécage, avec faculté de retrouver sa route en arrière comme en avant.

Tandis que dans tout autre domaine les vérités $<$ 'appuient $>$ et se rappellent les unes les autres à mesure qu'on avance, il semble qu'une fatalité veuille pour la langue que toute nouvelle vérité oblitère l'autre parce que les <vérités> initiales ne sont pas simples16 (195;ELG, p. 95-96).

Ce n'est pas un hasard si la métaphore de "pieu solide enfoncé dans le marécage » présente une similitude frappante avec un passage de K. Popper :

La science ne repose pas sur une base rocheuse. La structure audacieuse de ses théories s'édifie en quelque sorte sur un marécage. Elle est comme une construction bâtie sur pilotis. Les pieux sont enfoncés dans le marécage, mais pas jusqu'à la rencontre de quelque base naturelle ou « donnée » et, lorsque nous cessons d'essayer de les enfoncer davantage, [c'est] parce que nous sommes convaincus qu'ils sont assez solides pour supporter l'édifice, du moins provisoirement ${ }^{17}$.

16. La transcription proposée ici est différente de celle que donne l'édition de Gallimard, qui lit « une pièce solide » au lieu d' " un pieu solide ».

17. K. Popper, La Logique de la découverte scientifique, Paris, Payot, 1973, p. 111. 
La coïncidence est significative dans la mesure où Popper propose une épistémologie non fondationnelle pour laquelle la valeur de la vérité se mesure à la force heuristique. Mais, sceptique quant à la possibilité de la science du langage, Saussure écrit (fig. 8) :

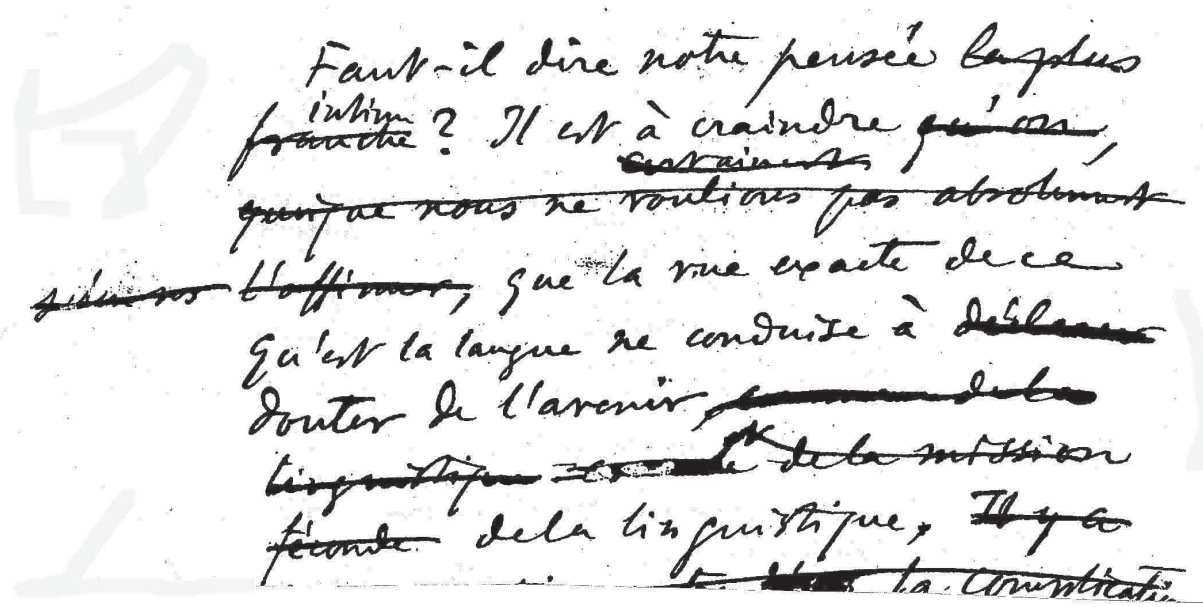

Fig. 8

Faut-il dire notre pensée taplus franehe <intime > ? Il est à craindre qu'on, qưique nuts ne voulions $<$ eertainement> pas absolument <selon nous $>$ l'affirmer, que la vue exacte de ce qu'est la langue ne conduise à déelarer douter de l'avenir [,eomme] de la linguistique [ill.] <et> de la mission féeonde de la linguistique (187; ELG, 29i(2), p. 87).

Rien n'illustre mieux le scepticisme de Saussure que cette «pensée intime ». La tension permanente entre l'idée de la science du langage que Saussure s'est prescrite à lui-même et le cercle herméneutique qui résiste à l'exposé linéaire ou déductif de l'édifice théorique n'a pu trouver son expression adéquate que dans un style aphoristique où l'on voit s'essayer et s'interrompre une pensée sans cesse retenue dans sa cristallisation momentanée. Notre interprétation de Saussure ne sortira pas indemne de cette confrontation vertigineuse avec ses manuscrits aphoristiques, qui la questionnent autant qu'elle les questionne. Du rayonnement du Mémoire de 1879 à l'écriture fragmentaire et nocturne prise dans le cercle herméneutique, c'est cette odyssée intellectuelle qui nous retient et nous étonne encore ${ }^{18}$.

18. Je remercie Madame Barbara Roth, conservatrice du département des Manuscrits de la Bibliothèque de Genève de l'aimable autorisation de publier le manuscrit de Saussure, ainsi que Mesdames Claire Fauvergue et Irène Fenoglio de leur relecture attentive. Je n'ai pu tenir compte, à mon grand regret, de l'édition de René Amacker (Science du langage, Genève, Droz) parue à la fin de l'année 2011, à un moment où le texte du présent article avait déjà été remis au comité de rédaction. 
Kazuhiro Matsuzawa est professeur à l'Université de Nagoya. Il a publié de nombreux ouvrages et articles parmi lesquels : « Notes pour un livre sur la linguistique générale. Présentation et édition », dans S. Bouquet (dir.), Éditions de l'Herne, coll. « Cahiers de l'Herne, $n^{\circ} 76$ », 2003, p. 319-322 ; «Le "décousu" du troisième cours de linguistique générale et le cercle herméneutique », dans Le Projet de Saussure, dir. J.-P. Bronckar, Droz, 2010, p. 61-78. Il prépare actuellement une édition critique d'inspiration génétique en japonais des Écrits de linguistique générale de Ferdinand de Saussure.

j46159a@piano.ocn.ne.jp

Résumés

\section{Puissance de l'écriture fragmentaire et « cercle vicieux »}

L'auteur se propose de montrer que l'écriture fragmentaire de Saussure dans les manuscrits dits De l'essence double du langage ouvre un nouvel espace de réflexion en mettant en question la prétention d'une pensée linéaire attachée à l'édifice théorique de la science du langage. Saussure se trouve emporté dans un « cercle vicieux » qui le fait remonter au présupposé au fur et à mesure où il avance. Prenant pour point de départ le lieu du langage ou bien la figure vocale, Saussure ne cesse d'en appeler au principe de la négativité différentielle en le présupposant. Mais ce principe n'est à l'œuvre que dans et par la conscience du sujet parlant, en dehors de laquelle le principe de la différence ferait de la langue un être ayant une existence indépendante de l'esprit humain. D'où l'hésitation de Saussure sur l'ordre de l'exposition. D'ailleurs à ce renvoi circulaire entre le lieu du langage, la négativité et la conscience du sujet s'oppose la dimension historique de la transmission de la langue. La tension permanente entre l'idée de la science du langage et le cercle herméneutique n'a pu trouver son expression adéquate que dans une écriture fragmentaire et inachevée.

The author sets out to show that Saussure's fragmentary writing in the manuscripts known as De l'essence double du langage opens a new field of reflection by challenging the claim of a linear thought bound up with the theory of the science of language. Saussure is drawn into a "vicious circle" that makes him return to the premise as he goes along. Starting from the place of language or the vocal figure Saussure constantly refers to the principle of differential negativity by presupposing it. But this principle is only at work in and by the speaking subject's consciousness, without which the principle of difference would make language a being existing independently of the human mind. Hence Saussure's hesitation on the order of the demonstration. Moreover this circular referral to the place of language, negativity and the subject's consciousness contradicts the historic dimension of the transmission of language. The permanent tension between the idea of the science of language and the hermeneutic circle could only be adequately expressed in a fragmentary, incomplete writing.
In diesem Text soll aufgezeigt werden, dass Saussures fragmentarische Schreibweise in den Manuskripten von De l'essence double du langage neue Denkanstösse schafft, da der Anspruch eines linearen Gedankens im theoretischen Gerüst der Sprachwissenschaft in Frage gestellt wird. Saussure befindet sich in einem „Teufelskreis“, der ihn, je weiter er vorankommt, zu den Grundlagen zurückführt. Ausgehend vom Ort der Sprache oder der Vokalfigur beruft sich Saussure unaufhörlich auf das Prinzip der differentiellen Negativität, das er als Voraussetzung betrachtet. Doch dieses Prinzip kommt nur im und durch das Bewusstsein des Sprechers zur Geltung; außerhalb dessen wäre die Sprache aufgrund des Prinzips der Differenz eine vom menschlichen Geist unabhängige Materie. Daher kommt Saussures Zögern bezüglich der Abfolge der Erläuterungen. Außerdem stellt sich diesem kreisförmigen Schwanken zwischen Ort der Sprache, Negativität und Bewusstsein des Sprechers die historische Dimension der Übermittlung der Sprache entgegen.

E1 autor se propone mostrar que la escritura fragmentaria de Saussure en los manuscritos llamados De la esencia doble del lenguaje abre un nuevo espacio de reflexión cuestionando la pretensión de un pensamiento lineal ligado al edificio teórico de la ciencia del lenguaje. Saussure se ve embarcado en un "círculo vicioso" que lo conduce a la presunción a medida que avanza. Tomando como punto de partida el lugar del lenguaje o la figura vocal, Saussure recurre permanentemente al principio de la negatividad diferencial presuponiéndolo. Pero este principio se manifiesta exclusivamente en (y por) la conciencia del sujeto hablante, al exterior de la cual el principio de la diferencia haría de la lengua un ser dotado de una existencia independiente del espíritu humano. ¿De dónde proviene la indecisión de Saussure con respecto al orden de la exposición? Además, a esa remisión circular entre el lugar del lenguaje, la negatividad y la conciencia del sujeto se opone la dimensión histórica de la transmisión de la lengua. La tensión permanente entre la idea de la ciencia del lenguaje y el círculo hermenéutico no pudo encontrar su expresión adecuada más que en una escritura fragmentaria e inacabada. 


\section{Résumés}

O autor pretende mostrar que a escrita fragmentária de Saussure nos manuscritos denominados Da dupla essência da linguagem abre um novo espaço de reflexão, por questionar a pretensão de um pensamento linear associado ao edifício teórico da ciência da linguagem. Saussure, à medida que avança, entra num "círculo vicioso" de retorno aos seus pressupostos. Quer parta do lugar da linguagem, quer da figura vocal, Saussure recorre constantemente ao princípio da negatividade diferencial, que pressupõe. Mas esse princípio opera exclusivamente em e através da consciência do sujeito falante, fora da qual o princípio diferencial tornaria a língua uma entidade com existência independente do espírito humano. Daí resulta a hesitação de Saussure quanto à ordem da exposição. Por outro lado, a esse reenvio circular entre o lugar da linguagem, a negatividade e a consciência do sujeito opõe-se a dimensão histórica da transmissão da língua. A tensão permanente entre a ideia da ciência da linguagem e o círculo hermenêutico encontra uma expressão adequada na escrita fragmentária e inacabada.
L'autore si propone di mostrare che la scrittura frammentaria di Saussure nei manoscritti De l' essence double du langage offre una nuova possibilità di riflessione mettendo in questione la pretesa di un pensiero lineare legato all'edificio teorico della scienza del linguaggio. Saussure si trova in un "circolo vizioso" che lo fa risalire al presupposto man mano che avanza. Prendendo il luogo del linguaggio - la figura vocale - come punto di partenza, Saussure non cessa di richiamarsi al principio della negatività differenziale nel presupporla. Ma tale principio non è all'opera che nella e per la coscienza del parlante, fuori dalla quale il principio della differenza farebbe della lingua un essere con un'esistenza indipendente dalla mente umana. Di qui viene l'esitazione di Saussure sull'ordine dell'esposizione. D'altra parte, a questa circolarità tra luogo del linguaggio, negatività e coscienza del soggetto si oppone la dimensione storica della trasmissione della lingua. La tensione permanente tra l'idea della scienza del linguaggio e il circolo ermeneutico non ha potuto trovare la sua espressione adeguata che in una scrittura frammentaria e non conclusa. 